\title{
A Stochastic MIMO Channel Model With Joint Correlation of Both Link Ends
}

\author{
Werner Weichselberger, Markus Herdin, Hüseyin Özcelik, Senior Member, IEEE, and \\ Ernst Bonek, Senior Member, IEEE
}

\begin{abstract}
This paper presents a novel stochastic channel model for multiple-input multiple-output (MIMO) wireless radio channels. In contrast to state-of-the-art stochastic MIMO channel models, the spatial correlation properties of the channel are not divided into separate contributions from transmitter and receiver. Instead, the joint correlation properties are modeled by describing the average coupling between the eigenmodes of the two link ends. The necessary and sufficient condition for the proposed model to hold is that the eigenbasis at the receiver is independent of the transmit weights, and vice versa. The authors discuss the mathematical elements of the model, which can be easily extracted from measurements, from a radio propagation point of view and explain the underlying assumption of the model in physical terms. The validation of the proposed model by means of measured data obtained from two completely different measurement campaigns reveals its ability to better predict capacity and spatial channel structure than other popular stochastic channel models.
\end{abstract}

Index Terms-Antenna arrays, channel capacity, channel modeling, MIMO channels, spatial diversity, spatial multiplexing.

\section{INTRODUCTION}

$\mathbf{M}$ ULTIPLE-INPUT multiple-output (MIMO) systems have recently attracted much attention. They promise a very high spectral efficiency because they allow the construction of parallel communication channels that are separated in the spatial domain. Under ideal conditions, the information theoretic capacity of a MIMO system grows linearly with the minimum of transmit and receive antennas [1], [2]. However, various measurements show that realistic MIMO channels show a significantly lower capacity [3]-[6]. This reduction of

Manuscript received August 22, 2003; revised July 12, 2004; accepted August 31,2004 . The editor coordinating the review of this paper and approving it for publication is D. I. Kim. This work was supported by Nokia Research Center, Radio Communications Laboratory, Nokia Group, Helsinki FIN-00045, Finland. This paper was presented in part at the 6th International Symposium on Wireless Personal Multimedia Communications, October 19-22, 2003, Yokosuka, Japan.

W. Weichselberger was with the Institut für Nachrichtentechnik und Hochfrequenztechnik (INTHF), Technische Universität Wien, Vienna, Austria. $\mathrm{He}$ is now with Woolf Solutions IT Consulting and Development, Vienna A-1230, Austria (e-mail: ww@woolf-solutions.at).

M. Herdin was with the Institut für Nachrichtentechnik und Hochfrequenztechnik (INTHF), Technische Universität Wien, Vienna, Austria. He is now with the Wireless Solution Laboratory, DoCoMo Communications Laboratories Europe GmbH, Munich D-80687, Germany (e-mail: herdin@ docomolab-euro.com).

H. Özcelik and E. Bonek are with the Institut für Nachrichtentechnik und Hochfrequenztechnik (INTHF), Technische Universität Wien, Vienna A-1040, Austria (e-mail: huseyin.ozcelik@nt.tuwien.ac.at; ernst.bonek@nt. tuwien.ac.at).

Digital Object Identifier 10.1109/TWC.2005.858030 capacity is due to the spatial correlation of the MIMO channel coefficients [7], [8].

Besides geometrical approaches like, e.g., [9], many publications dealing with MIMO channel modeling aim at describing the spatial correlation properties of MIMO channels directly, e.g., [4], [5], [10]-[13]. Their common approach is to model the correlation at the receiver and at the transmitter independently, neglecting the statistical interdependence of both link ends. The authors of [3] and [14] have shown that realistic indoor MIMO channels cannot be modeled adequately by this approach; channel capacity is underestimated when the number of antennas at one link end becomes larger than two or three.

Another question is whether aggregate statistical parameters like ergodic capacity and eigenvalue distributions alone are sufficient to judge whether a model is appropriate or not. Based on evidence presented in [14] and [15], we strongly believe it is not. Aggregate metrics provide necessary but not sufficient conditions for the validation of channel models. Eventually, the ultimate goal of MIMO channel modeling is to reflect the underlying spatial structure of the radio environment. An interesting approach in this respect, called "virtual channel representation," has been presented in [16]. Its author utilizes a virtual partitioning of the spatial domain to characterize the MIMO channel. This partitioning is fixed and predefined, which poses some difficulties with respect to the rendering of measured channels.

Due to aspects of practicability, a MIMO channel model is subject to some restrictions. A small number of modeling parameters are desirable. It facilitates the application of the model and reduces estimation errors when it comes to estimating the model parameters from measurements. A concise mathematical description is necessary for practicable analytical investigations of the MIMO channel, e.g., derivation of optimum transmit or coding strategies. Instead of designing a specific model for each type of environment, we aim at a rather general model covering most real-world scenarios. Furthermore, the parameters of the model should be interpretable with respect to wave propagation, and we would like to be able to parameterize the model directly from measurements or channel estimates obtained during system operation.

We will present a novel stochastic MIMO channel model ${ }^{1}$ that has been inspired by the correlation-based models, e.g., the "Kronecker model" [4], as well as by the "virtual channel representation" of [16]. Combining the advantages of both

\footnotetext{
${ }^{1}$ The basic idea of this model has already been presented in [17].
} 
approaches, it shows enhanced capabilities to model the spatial multipath structure of realistic MIMO channels correctly. It does not only account for the correlation at both link ends (as does the Kronecker model) but also models their mutual dependence (as does the "virtual channel representation"). Furthermore, its mathematical description is simple and concise. Independently, a similar modeling approach has been introduced in [18]-[20]. In contrast to our present work, the channel modeling perspective was not the focus of these papers.

Validating a channel model with a single measurement campaign does not allow for verifying the generality of the model. In order to avoid the pitfall of finding an environment that fits the proposed model, we will present validation results from two different measurement campaigns: an indoor office scenario and a suburban outdoor area.

The rest of the paper is organized as follows. In the next section, we will present the signal model and some formal definitions. In Section III, we will shortly discuss the restrictions of the "Kronecker model" and the "virtual channel representation." The new channel model is presented and discussed in Section IV. Finally, in Section V, we will evaluate the performance of the new model by means of measured MIMO impulse responses.

\section{Signal Model AND Definitions}

The presented model focuses on the spatial structure of MIMO channels. We will, therefore, restrict our considerations to stationary and frequency-flat channels that can be described by a random channel matrix $\boldsymbol{H}$ subject to small-scale fading. For reasons of clarity, we will assume in the following the channel matrix $\boldsymbol{H}$ to be multivariate complex-normal distributed with zero mean, i.e., Rayleigh fading.

Denoting the number of receive (Rx) and transmit (Tx) antennas in the forward link with $M_{A}$ and $M_{B}$, respectively, the matrix $\boldsymbol{H}$ is of size $M_{A} \times M_{B}$. The signal vector $\boldsymbol{y}$ at the $M_{A}$ receive antennas reads as $\boldsymbol{y}=\boldsymbol{H} \boldsymbol{x}+\boldsymbol{n}$, where $\boldsymbol{x}$ denotes the transmit signal vector and $\boldsymbol{n}$ is the noise vector observed at the receiver. Utilizing the channel in the reverse link, the link ends switch role with respect to transmitting and receiving the radio signal. In order to avoid confusion, we will denote the link ends with the labels "A" and "B," respectively. Both link ends A and B can act as receiver or transmitter depending on the link direction. Throughout the entire paper, the subscripts $\bullet_{\mathrm{A}}$ and $\bullet_{\mathrm{B}}$ will indicate to which side of the radio link an entity is associated.

We will use the $\operatorname{vec}(\bullet)$ operator that stacks the columns of a matrix into one tall vector, the $\operatorname{diag}(\bullet)$ operator that puts the elements of a $1 \times M$ vector onto the main diagonal of an $M \times M$ diagonal matrix, and the $\operatorname{tr}(\bullet)$ operator that denotes the trace of a matrix. The superscripts $\bullet^{\mathrm{T}}, \bullet^{\mathrm{H}}$, and $\bullet *$ denote the matrix transpose, conjugate matrix transpose, and the complex conjugate, respectively. Finally, we define two matrix product operators. The symbol $\otimes$ denotes the Kronecker matrix product and $\odot$ denotes the element-wise product of two matrices.

The total mean energy of the channel will be denoted by $P_{\boldsymbol{H}} \triangleq \mathrm{E}_{\boldsymbol{H}}\left\{\operatorname{tr}\left(\boldsymbol{H} \boldsymbol{H}^{\mathrm{H}}\right)\right\}$. Here, and for all subsequent considerations, the expectation operator is performed with respect to different channel realizations of one and the same scenario. We define a scenario as a region in time and space for which the channel statistics are approximately constant.

\section{A. Correlation Properties of MIMO Channel}

In order to describe the spatial behavior of a general MIMO channel, a full correlation matrix that specifies the $M_{A} M_{B} \times$ $M_{A} M_{B}$ mutual correlation values between all channel matrix elements is required. In accordance to the literature, we will use

$$
\boldsymbol{R}_{\boldsymbol{H}} \triangleq \mathrm{E}_{\boldsymbol{H}}\left\{\operatorname{vec}(\boldsymbol{H}) \operatorname{vec}(\boldsymbol{H})^{\mathrm{H}}\right\}
$$

for the description of the joint correlation properties of both link ends.

Additionally, we will use "one-sided" correlation matrices that denote the spatial correlations of one link end only. Because the two link ends of a MIMO channel cannot be considered as independent, the one-sided correlation matrices have to be parameterized by the statistical signal properties of the other link end

$$
\boldsymbol{R}_{\mathrm{A}, \boldsymbol{Q}_{\mathrm{B}}} \triangleq \mathrm{E}_{\boldsymbol{H}}\left\{\boldsymbol{H} \boldsymbol{Q}_{\mathrm{B}} \boldsymbol{H}^{\mathrm{H}}\right\} \text { and } \boldsymbol{R}_{\mathrm{B}, \boldsymbol{Q}_{\mathrm{A}}} \triangleq \mathrm{E}_{\boldsymbol{H}}\left\{\boldsymbol{H}^{\mathrm{T}} \boldsymbol{Q}_{\mathrm{A}} \boldsymbol{H}^{*}\right\}
$$

where $Q_{\mathrm{A}}$ and $\boldsymbol{Q}_{\mathrm{B}}$ are the spatial signal covariance matrices of sides $\mathrm{A}$ and $\mathrm{B}$, respectively.

The unparameterized one-sided correlation matrices $\boldsymbol{R}_{\mathrm{A}} \triangleq$ $\mathrm{E}_{\boldsymbol{H}}\left\{\boldsymbol{H} \boldsymbol{H}^{\mathrm{H}}\right\}$ and $\boldsymbol{R}_{\mathrm{B}} \triangleq \mathrm{E}_{\boldsymbol{H}}\left\{\boldsymbol{H}^{\mathrm{T}} \boldsymbol{H}^{*}\right\}$, which are well established in the literature, are obtained by assuming the signal covariance of the other link end to be spatially white. Their eigenbases will be denoted by $\boldsymbol{U}_{\mathrm{A}}$ and $\boldsymbol{U}_{\mathrm{B}}$, respectively, and consist of the eigenvectors denoted by $\boldsymbol{u}_{\mathrm{A}, m}$ and $\boldsymbol{u}_{\mathrm{B}, n}$, respectively. Furthermore, we define the matrices $\Lambda_{\mathrm{A}}$ and $\Lambda_{\mathrm{B}}$ as diagonal matrices containing the eigenvalues of $\boldsymbol{R}_{\mathrm{A}}$ and $\boldsymbol{R}_{\mathrm{B}}$, respectively, as well as the vectors $\tilde{\lambda}_{\mathrm{A}}$ and $\tilde{\lambda}_{\mathrm{B}}$ to consist of the square roots of the eigenvalues of $\boldsymbol{R}_{\mathrm{A}}$ and $\boldsymbol{R}_{\mathrm{B}}$, respectively.

\section{Discussion of Two Existing Channel Models}

A significant drawback of the full correlation matrix is its huge size. It requires $\left(M_{\mathrm{B}} M_{\mathrm{A}}\right)^{2}$ real-valued parameters to be fully specified. Moreover, a direct interpretation of the elements of $\boldsymbol{R}_{\boldsymbol{H}}$ with respect to the physical propagation of the radio channel is difficult. In the literature, this problem is mainly addressed by two analytical channel model approaches: the "Kronecker model," e.g., [4], [5], [10]-[13], and the "virtual channel representation" presented in [16].

Both models imply severe restrictions on the structure of the MIMO channel. In the following, we will shortly discuss these restrictions by means of the parameterized one-sided correlation matrices $\boldsymbol{R}_{\mathrm{A}, \boldsymbol{Q}_{\mathrm{B}}}$ and the following theorem.

Theorem 1: For a MIMO channel matrix that is modeled as

$$
\boldsymbol{H}=\boldsymbol{B}_{\mathrm{A}}\left(\left(\sum_{k=1}^{K} \tilde{\boldsymbol{\lambda}}_{\mathrm{A}, k} \tilde{\boldsymbol{\lambda}}_{\mathrm{B}, k}^{\mathrm{T}}\right) \odot \boldsymbol{G}\right) \boldsymbol{B}_{\mathrm{B}}^{\mathrm{T}}
$$


(where $\boldsymbol{B}_{A}$ and $\boldsymbol{B}_{\mathrm{B}}$ are unitary matrices; $\boldsymbol{G}$ is an independent and identically distributed (i.i.d.) random matrix with zero mean and unit variance elements; and the vectors $\tilde{\boldsymbol{\lambda}}_{\mathrm{A}, k}$ and $\tilde{\lambda}_{\mathrm{B}, k}$ are real valued) the resulting parameterized one-sided correlation matrices $\boldsymbol{R}_{\mathrm{A}, \boldsymbol{Q}_{\mathrm{B}}}$ and $\boldsymbol{R}_{\mathrm{B}, \boldsymbol{Q}_{\mathrm{A}}}$ are given as

$$
\begin{aligned}
& \boldsymbol{R}_{\mathrm{A}, \boldsymbol{Q}_{\mathrm{B}}}=\boldsymbol{B}_{\mathrm{A}}\left(\sum_{k=1}^{K} \Lambda_{\mathrm{A}, k} \operatorname{tr}\left(\Lambda_{\mathrm{B}, k} \boldsymbol{B}_{\mathrm{B}}^{\mathrm{T}} \boldsymbol{Q}_{\mathrm{B}} \boldsymbol{B}_{\mathrm{B}}^{*}\right)\right) \boldsymbol{B}_{\mathrm{A}}^{\mathrm{H}} \\
& \boldsymbol{R}_{\mathrm{B}, \boldsymbol{Q}_{\mathrm{A}}}=\boldsymbol{B}_{\mathrm{B}}\left(\sum_{k=1}^{K} \Lambda_{\mathrm{B}, k} \operatorname{tr}\left(\Lambda_{\mathrm{A}, k} \boldsymbol{B}_{\mathrm{A}}^{\mathrm{T}} \boldsymbol{Q}_{\mathrm{A}} \boldsymbol{B}_{\mathrm{A}}^{*}\right)\right) \boldsymbol{B}_{\mathrm{B}}^{\mathrm{H}}
\end{aligned}
$$

where $\boldsymbol{\Lambda}_{\mathrm{A}, k} \triangleq \operatorname{diag}\left(\tilde{\boldsymbol{\lambda}}_{\mathrm{A}, k}\right)^{2}$ and $\boldsymbol{\Lambda}_{\mathrm{B}, k} \triangleq \operatorname{diag}\left(\tilde{\boldsymbol{\lambda}}_{\mathrm{B}, k}\right)^{2} \quad$ (see proof in the Appendix).

\section{A. Kronecker Model}

The Kronecker model neglects the joint spatial structure and describes the MIMO channel by the separated correlation properties of both link ends

$$
\boldsymbol{H}_{\mathrm{kron}}=\frac{1}{P_{\boldsymbol{H}}} \boldsymbol{R}_{\mathrm{A}}^{\frac{1}{2}} \boldsymbol{G}\left(\boldsymbol{R}_{\mathrm{B}}^{\frac{1}{2}}\right)^{\mathrm{T}}
$$

where $G$ is an i.i.d. random matrix whose entries are zeromean complex-normal distributed. Equation (2) can be equivalently formulated as $\boldsymbol{H}_{\text {kron }}=\left(1 / P_{\boldsymbol{H}}\right) \cdot \boldsymbol{U}_{\mathrm{A}}\left(\left(\tilde{\boldsymbol{\lambda}}_{\mathrm{A}} \tilde{\boldsymbol{\lambda}}_{\mathrm{B}}^{\mathrm{T}}\right) \odot \boldsymbol{G}\right) \boldsymbol{U}_{\mathrm{B}}^{\mathrm{T}}$. Thus, we can conclude from Theorem 1 that $\boldsymbol{R}_{\mathrm{A}, \boldsymbol{Q}_{\mathrm{B}}}=c \boldsymbol{R}_{\mathrm{A}}$, where $c$ is a real-valued and nonnegative scalar scaling factor.

The "Kronecker model" implies that the transmitter does not effect the spatial properties of the received signal at all. We will see in Section V how that implication impairs the ability of the "Kronecker model" to render the spatial structure of measured MIMO channels.

\section{B. Virtual Channel Representation}

The "virtual channel representation" can be written as

$$
\boldsymbol{H}_{\mathrm{virt}}=\boldsymbol{A}_{\mathrm{A}}\left(\tilde{\boldsymbol{\Omega}}_{\mathrm{virt}} \odot \boldsymbol{G}\right) \boldsymbol{A}_{\mathrm{B}}^{\mathrm{T}}
$$

where $G$ is again an i.i.d. random matrix whose entries are zero-mean complex-normal distributed. The matrices $\boldsymbol{A}_{\mathrm{A}}$ and $\boldsymbol{A}_{\mathrm{B}}$ are channel independent discrete Fourier transform (DFT) matrices of size $M_{\mathrm{A}} \times M_{\mathrm{A}}$ and $M_{\mathrm{B}} \times M_{\mathrm{B}}$, respectively. By means of Theorem 1, we conclude that $\boldsymbol{R}_{\mathrm{A}, \boldsymbol{Q}_{\mathrm{B}}}=$ $\boldsymbol{A}_{\mathrm{A}} \boldsymbol{\Lambda}_{\mathrm{A}, \boldsymbol{Q}_{\mathrm{B}}}^{\text {(vit) }} \boldsymbol{A}_{\mathrm{B}}^{\mathrm{T}}$, where the real-valued diagonal matrix $\boldsymbol{\Lambda}_{\mathrm{A}, \boldsymbol{Q}_{\mathrm{B}}}^{\text {(virt) }}$ depends on the spatial distribution of the transmit signal.

The "virtual channel representation" restricts the eigenbases of one-sided correlation matrices to predefined DFT matrices. Furthermore, as pointed out in [16], the "virtual channel representation" is restricted to single polarized uniform linear arrays (ULAs).

It is shown in [16] that the channel matrix elements of a ULA form a segment of a two-dimensional (2-D) stationary process. Thus, the DFT matrices serve as asymptotically (as the number of antenna elements goes to infinity) optimal eigenfunctions for the channel matrix, and the elements of the fading matrix
$\left(\tilde{\boldsymbol{\Omega}}_{\mathrm{virt}} \odot \boldsymbol{G}\right)$ constitute samples of the underlying spectral representation and are hence uncorrelated.

However, for a practical number of antenna elements, the approximation of the true eigenbases by the predefined DFT matrices can be rather poor. As a consequence, the matrix elements of $\boldsymbol{G}$ in (3) can become significantly correlated when "virtual channel representation" is applied to measured MIMO channels, as shown in Section V.

\section{Novel Stochastic MiMO Channel Model}

\section{A. Modeling Assumption}

The purpose of the new model is threefold. We want to

- alleviate the restriction imposed by the "Kronecker model" and describe the joint spatial structure of the channel as the "virtual channel representation" does;

- alleviate the restriction imposed by the "virtual channel representation" and adopt the spatial eigenbases to the channel as well as to the array configuration (including array geometry, element characteristics and element polarizations) as the "Kronecker model" does;

- formulate a model that includes both the "Kronecker model" and the "virtual channel representation" as special cases.

To these ends, we postulate the following modeling assumption.

All one-sided spatial correlation matrices of link end A (B) have the same eigenbasis that is defined by the unparameterized one-sided correlation matrix $\boldsymbol{R}_{\mathrm{A}}\left(\boldsymbol{R}_{\mathrm{B}}\right)$. Their eigenvalues may differ.

In formal notation, this assumption reads as

$$
\boldsymbol{R}_{\mathrm{A}, \boldsymbol{Q}_{\mathrm{B}}}=\boldsymbol{U}_{\mathrm{A}} \Lambda_{\mathrm{A}, \boldsymbol{Q}_{\mathrm{B}}} \boldsymbol{U}_{\mathrm{A}}^{\mathrm{H}} \quad \text { and } \quad \boldsymbol{R}_{\mathrm{B}, \boldsymbol{Q}_{A}}=\boldsymbol{U}_{\mathrm{B}} \Lambda_{\mathrm{B}, \boldsymbol{Q}_{\mathrm{A}}} \boldsymbol{U}_{\mathrm{B}}^{\mathrm{H}}
$$

where $\boldsymbol{\Lambda}_{\mathrm{A}, \boldsymbol{Q}_{\mathrm{B}}}$ and $\boldsymbol{\Lambda}_{\mathrm{B}, \boldsymbol{Q}_{\mathrm{A}}}$ are real-valued diagonal matrices with nonnegative entries.

The eigenbases do not depend on the signal correlation of the other link end (they do, however, depend on the channel and the array configuration). The eigenvalues do, in general, differ for each transmit signal correlation matrix.

\section{B. Physical Interpretation of the Modeling Assumption}

What are the implications of the modeling assumption in physical terms? The spatial correlation of transmit weights determines how much power is radiated into which directions (and polarizations). According to the assumption made, the spatial eigenbases are not affected by the transmit weights and, thus, reflect the radio environment only, i.e., number, positions, and strengths of the scatterers.

The eigenvalues, on the other hand, do depend on the transmit weights. They reflect how the scatterers are illuminated by the radio waves propagating from the transmitter. Radiating in certain directions, for example, may illuminate only certain scatterers and leave others "dark." Of course, the eigenvalues are additionally influenced by the radio environment as well. 


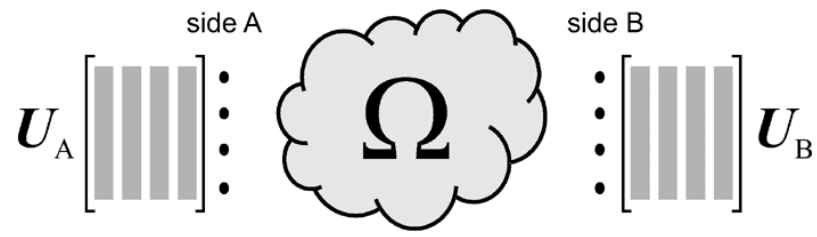

Fig. 1. Parameters required for the new model. The spatial eigenbasis $\boldsymbol{U}_{\mathrm{A}}$ at side A, the spatial eigenbasis $\boldsymbol{U}_{\mathrm{B}}$ at side B, and the coupling matrix $\boldsymbol{\Omega}$.

By means of the given physical interpretation, it becomes obvious that the presented modeling assumption holds only approximately. It is well known [21] that the spatial eigenbases are influenced by the spatial structure of the transmit signals as well. However, we want to stress that the presented assumption is less restrictive and provides a better approximation of physical reality than both the "Kronecker model" and the "virtual channel representation."

\section{Formulation of Channel Model}

According to Theorem 1, the new channel model resulting from modeling assumption (4) can be written as

$$
\boldsymbol{H}_{\text {model }}=\boldsymbol{U}_{\mathrm{A}}(\tilde{\boldsymbol{\Omega}} \odot \boldsymbol{G}) \boldsymbol{U}_{\mathrm{B}}^{\mathrm{T}}
$$

where $G$ is a random matrix with i.i.d. zero-mean complexnormal entries with unit variance; and $\tilde{\boldsymbol{\Omega}}$ has, in general, full rank and consists of real-valued nonnegative elements. By little massaging of (5), we can calculate $\tilde{\boldsymbol{\Omega}}$ as the element-wise square root of the coupling matrix $\Omega$ given by

$$
[\boldsymbol{\Omega}]_{m, n}=\omega_{m, n}=\mathrm{E}_{\boldsymbol{H}}\left\{\left|\boldsymbol{u}_{\mathrm{A}, m}^{\mathrm{H}} \boldsymbol{H} \boldsymbol{u}_{\mathrm{B}, n}^{*}\right|^{2}\right\} .
$$

We call $\Omega$ "coupling matrix" because its coefficients specify the mean amount of energy that is coupled from the $m$ th eigenvector of side-A to the $n$th eigenvector of side $\mathrm{B}$ (or vice versa).

All we need for modeling the spatial properties of a MIMO channel are (cf. Fig. 1)

- the spatial eigenbasis $U_{\mathrm{A}}$ at side A;

- the spatial eigenbasis $U_{\mathrm{B}}$ at side B;

- the average energy of the virtual single-input single-output (SISO) channel between each eigenmode of side A and each eigenmode of side $\mathrm{B}, \boldsymbol{\Omega}$, linking the correlation properties of both ends.

1) Full Correlation Matrix: Denoting the elements of $\boldsymbol{G}$ with $g_{n, m}$ and writing $\boldsymbol{H}_{\text {model }}$ as a sum of all eigenmodes, we can stack the channel matrix $\boldsymbol{H}_{\text {model }}$ as

$$
\begin{aligned}
\operatorname{vec}\left(\boldsymbol{H}_{\text {model }}\right) & =\sum_{m=1}^{M_{\mathrm{A}}} \sum_{n=1}^{M_{\mathrm{B}}} \operatorname{vec}\left(\sqrt{\omega_{m, n}} g_{m, n} \boldsymbol{u}_{\mathrm{A}, m} \boldsymbol{u}_{\mathrm{B}, n}^{\mathrm{T}}\right) \\
& =\sum_{m=1}^{M_{\mathrm{A}}} \sum_{n=1}^{M_{\mathrm{B}}} \sqrt{\omega_{m, n}} g_{m, n}\left(\boldsymbol{u}_{\mathrm{B}, n} \otimes \boldsymbol{u}_{\mathrm{A}, m}\right)
\end{aligned}
$$

and calculate the full correlation matrix of $\boldsymbol{H}_{\text {model }}$ as

$$
\begin{aligned}
\boldsymbol{R}_{\boldsymbol{H}, \text { model }} & =\sum_{m=1}^{M_{\mathrm{A}}} \sum_{n=1}^{M_{\mathrm{B}}} \omega_{m, n}\left(\boldsymbol{u}_{\mathrm{B}, n} \otimes \boldsymbol{u}_{\mathrm{A}, m}\right)\left(\boldsymbol{u}_{\mathrm{B}, n} \otimes \boldsymbol{u}_{\mathrm{A}, m}\right)^{\mathrm{H}} \\
& =\sum_{m=1}^{M_{\mathrm{A}}} \sum_{n=1}^{M_{\mathrm{B}}} \omega_{m, n}\left(\boldsymbol{u}_{\mathrm{B}, n} \boldsymbol{u}_{\mathrm{B}, n}^{H}\right) \otimes\left(\boldsymbol{u}_{\mathrm{A}, m} \boldsymbol{u}_{\mathrm{A}, m}^{\mathrm{H}}\right)
\end{aligned}
$$

It is interesting to note that the full correlation matrix shows a Kronecker structure on eigenmode level. Equation (6) provides the eigendecomposition of $\boldsymbol{R}_{\boldsymbol{H}}$ with $\left(\boldsymbol{u}_{\mathrm{B}, n} \otimes \boldsymbol{u}_{\mathrm{A}, m}\right)$ as eigenvectors and $\omega_{m, n}$ as eigenvalues.

The informed reader will notice that this section has been partly inspired by [16] and the "Kronecker model." Specifically, the new model reduces to the "virtual channel representation" by forcing the eigenbases to be DFT matrices; and it reduces the "Kronecker model" by forcing the coupling matrix $\Omega$ to be of rank one.

\section{Structure of Coupling Matrix}

We want to point out that the eigenvalues $\lambda_{\mathrm{B}, n}$ and $\lambda_{\mathrm{A}, m}$ of the one-sided correlation matrices $\boldsymbol{R}_{\mathrm{B}}$ and $\boldsymbol{R}_{\mathrm{A}}$ do not directly influence the model (5). They are given implicitly by the elements of $\boldsymbol{\Omega}$, i.e.,

$$
\lambda_{\mathrm{A}, m}=\sum_{n=1}^{M_{\mathrm{B}}} \omega_{m, n} \quad \text { and } \quad \lambda_{\mathrm{B}, n}=\sum_{m=1}^{M_{\mathrm{A}}} \omega_{m, n} .
$$

As already observed in [16], the structure of $\Omega$ reflects the spatial arrangement of scattering objects and influences the capacity as well as the degree of diversity that is experienced on spatially multiplexed channels. It tells us how many parallel data streams can be multiplexed, which degree of diversity is present at side $\mathrm{A}$ and at side $\mathrm{B}$, and how much beamforming gain can be achieved. Fig. 2 shows some examples of structures of $\boldsymbol{\Omega}$ and corresponding physical radio environments. In order to aid intuition, we will, in the following, think of side $\mathrm{A}$ as the receiving link end and of side $\mathrm{B}$ as the transmitter.

As defined in the previous section, $\omega_{m, n}$ denotes the average power of the virtual SISO channel between the $m$ th eigenmode of side A and the $n$th eigenmode of side B. A nonzero element of $\Omega$ establishes a link between the respective eigenmodes. A zero element of the coupling matrix means that the respective eigenmodes do not couple into each other. In Fig. 2, the elements of $\Omega$ are depicted as gray (significant power) and white (no power) squares. The right hand side of Fig. 2 simplifies the eigenmodes to discrete directions. Such an interpretation of eigenmodes is not correct in general, but it facilitates their visualization. ${ }^{2}$ Think of an eigenmode as the unrestricted antenna pattern generated by this mode. The number of eigenmodes present in the channel considered equals the number of

\footnotetext{
${ }^{2}$ We want to emphasize that the proposed model is not restricted to a directional interpretation of eigenmodes.
} 

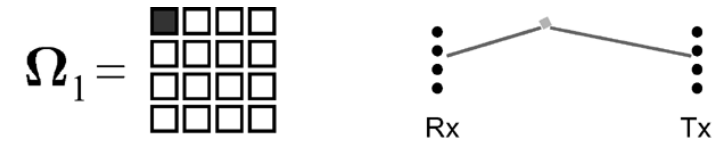

$\Omega_{2}=$ 㗊㗊

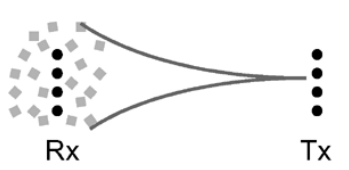

$\Omega_{3}=$ 㗊

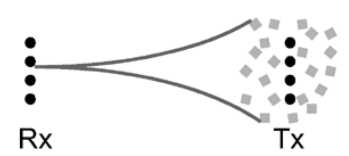

$\Omega_{4}=$ 㗊㗊

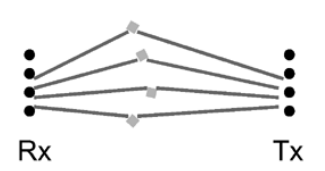

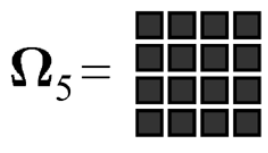

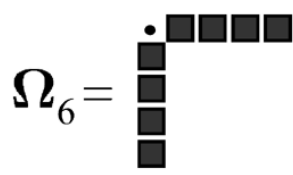
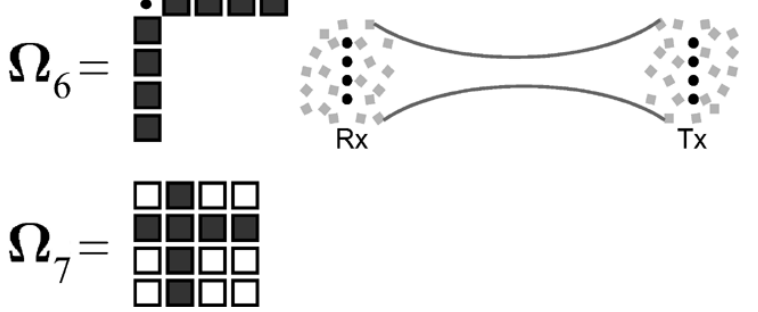

Fig. 2. Various structures of the coupling matrix $\Omega$ (gray squares: significant power; blank squares: no power; $\boldsymbol{\Omega}_{6}$ is the outer product of two vectors) and corresponding radio environments (black dots: antenna array; gray squares: scatterers).

resolvable multipath components, which evidently is a lower bound to all multipath components present.

The first example $\Omega_{1}$ has only a single nonzero element of the coupling matrix. This means that a single resolvable multipath component is present in the channel. Whether this is a line of sight (LOS) component or due to a scattering object is not determined by $\boldsymbol{\Omega}$. A single multipath component offers no diversity and does not support spatial multiplexing. On the other hand, the concentration of channel energy to a single $\mathrm{Rx}$ and a single Tx eigenmode allows for the enhancement of signal power by means of beamforming. Based on statistical knowledge only, we can focus the Rx and Tx antenna pattern onto the signal space of rank one and achieve a beamforming gain of $M_{\mathrm{A}} M_{\mathrm{B}}$.

The second example $\Omega_{2}$ shows a fully loaded single column of the coupling matrix. From the viewpoint of the Tx link end, this channel behaves the same as $\boldsymbol{\Omega}_{1}$ : it sees only a single spatial channel component, which does not support diversity. However, from the viewpoint of the Rx link end, the situation is different: at least $M_{\mathrm{A}}$ independent multipath components arrive at the Rx antenna, causing a spatial diversity of order $M_{\mathrm{A}}$. The physical explanation for the full channel rank at the Rx side is a locally rich scattering environment, while the rank-one channel of the Tx side can have two reasons. Either the size of the Rx scattering cluster is much smaller than its distance (depicted in Fig. 2), which causes the Tx array responses of all multipath components to be approximately identical. Or there is no LOS between the Rx cluster and the Tx array, and all multipath components travel from the Tx array via the same scattering object to the Rx side. While the Rx side offers spatial diversity, the concentration of channel energy onto a single Tx eigenmode allows for a Tx beamforming gain of $M_{\mathrm{B}}$ without instantaneous channel knowledge.

The third example $\boldsymbol{\Omega}_{3}$ is the same as $\boldsymbol{\Omega}_{2}$ with sides A and B interchanged. Because the channels of the first three examples collapse to a SISO, single-input multiple-output (SIMO), or multiple-input single-output (MISO) channel, none of these exemplary channels can support spatial multiplexing.

A strictly diagonal coupling matrix is depicted by $\boldsymbol{\Omega}_{4}$. Because the ordering of the Rx and Tx eigenmodes is arbitrary, this example is equivalent to any channel that shows a single entry on each row and column of $\boldsymbol{\Omega}$. The diagonal structure tells us that each Tx eigenmode is strictly linked to a single $\mathrm{Rx}$ eigenmode. Such a linkage could be via LOS, a single scatterer, or a scattering cluster. The depicted physical example shows single scatterers as the cause for the diagonal entries. The number of independent multipath components, and thus the order of diversity, is limited by the smaller antenna array, i.e., $\min \left(M_{\mathrm{A}}, M_{\mathrm{B}}\right)$. In contrast to $\boldsymbol{\Omega}_{2}$ and $\boldsymbol{\Omega}_{3}$, the diversity is not associated with a single link end but is a joint $\mathrm{Rx}-\mathrm{Tx}$ diversity. The transmitter has to span the entire possible signal space in order to enable full Rx diversity. A limited rank of the Tx signal covariance would directly lead to a reduced diversity at the receiver. Beamforming based on statistical knowledge is possible neither at the Rx side nor at the Tx side as the channel appears as fully decorrelated at both link ends. $\boldsymbol{\Omega}_{4}$ is the first example that supports spatial multiplexing. It does so in a very convenient way. By transmitting a separate data stream on each Tx eigenmode, the parallel data streams arrive on orthogonal $\mathrm{Rx}$ eigenmodes. The channel behaves as $\min \left(M_{\mathrm{A}}, M_{\mathrm{B}}\right)$ nicely separated and parallel SISO channels.

Example $\boldsymbol{\Omega}_{5}$ shows a fully loaded structure of the coupling matrix. Each Tx eigenmode is linked with each Rx eigenmode. If all elements of $\boldsymbol{\Omega}_{5}$ are exactly identical, this is the spatially white MIMO case. Note that, for a MIMO channel to be spatially white, it does not suffice to cause spatially white Rx and Tx sides, like, e.g., $\boldsymbol{\Omega}_{4}$. In the ideal spatially white case, the channel matrix $\boldsymbol{H}$ is an i.i.d. random matrix, which is well known to support full spatial multiplexing and full diversity. ${ }^{3} \mathrm{~A}$ possible physical environment leading to $\Omega_{5}$ is a rich scattering cluster containing both link ends.

We can identify a special case of $\boldsymbol{\Omega}_{5}$ depicted by $\boldsymbol{\Omega}_{6}$. It shows a coupling matrix of rank one, i.e., it is the outer product of a Rx-related and a Tx-related power distribution. This special case is identical to the "Kronecker model." Such a spatial channel characteristic can occur when scatterer clusters around the link ends are far apart.

\footnotetext{
${ }^{3}$ If the elements of $\boldsymbol{\Omega}$ are of the same order of magnitude, this holds true approximately.
} 
However, in [3] and [14], it was shown that the "Kronecker model" lacks essential degrees of freedom for general MIMO channels as all $M_{\mathrm{A}} \cdot M_{\mathrm{B}}$ elements of $\boldsymbol{\Omega}_{\mathrm{kron}}$ are determined by means of $M_{\mathrm{A}}+M_{\mathrm{B}}$ eigenvalues. It cannot generate diagonally dominated coupling matrices, the elements of $\Omega$ are as evenly distributed as possible. This leads to wrong capacity estimates and a mismatch of the modeled and measured multipath structure.

The last example $\boldsymbol{\Omega}_{7}$ is a rather unusual case. Such a crosslike structure leads to full rank correlation matrices at both link ends. Nevertheless, an instantaneous channel realization will have the same cross-shape (in the eigendomain) and, thus, its rank will be strictly limited to 2 . The cross-like structure behaves in some sense like a keyhole channel, which is described in, e.g., [11], [22], and [23]. However, the mechanism that leads to a rank deficiency of instantaneous realizations is different. Specifically, the cross-shape can be identified by knowledge of the MIMO channel correlation matrix, whereas the keyhole phenomenon does not appear in the channel correlation. $\boldsymbol{\Omega}_{7}$ should serve as a clear example that it is not possible to derive the joint MIMO structure by knowledge of the link ends only.

Two entities are of great interest with respect to MIMO channels: diversity and mutual information. The total degree of spatial diversity present in the channel is given by the number of independently fading channel components, which is identical to the number of nonzero eigenvalues of $\boldsymbol{R}_{\boldsymbol{H}}$. According to (6), this number is given by the number of nonzero elements of $\boldsymbol{\Omega}$.

In line with the discussion of Fig. 2, we can state that the capacity of examples 1 to 3 (no multiplexing) is smaller than the capacity of example 7 (limited to rank two), which is in turn smaller than the capacity of examples 4 to 6 (full multiplexing). Comparing the capacity of examples 4 to 6 is not straightforward. Inspection of various simulations with synthetic and measured (cf. Section V) MIMO channels suggests the following rule of thumb: the more diagonal the structure of $\Omega$ (while the total channel power is constant), the higher the mutual information. Having the eigenvalues of the correlation matrices of sides $\mathrm{A}$ and $\mathrm{B}$ as given side constraints, the Kronecker model $\boldsymbol{\Omega}_{6}$ exhibits the least diagonal structure. The "Kronecker model" underestimated channel capacity for all measurement results of Section V. Concerning analytical results on the capacity of MIMO channels like $\boldsymbol{\Omega}_{4}, \boldsymbol{\Omega}_{5}$, and $\boldsymbol{\Omega}_{6}$, we want to refer the reader to [24]. There, upper and lower bounds on capacity and asymptotic capacity results for infinitely large antenna arrays are provided for the virtual channel representation.

\section{VALidation of Mimo Channel Model With MEASURED DATA}

In order to avoid the pitfall of finding an environment that fits the proposed model, we present validation results from two completely different measurement campaigns. One was conducted in the premises of an office building, the other one was carried out in a suburban outdoor environment. The antenna configurations were different, the carrier frequency was different, as were the people performing the measurements.

We will utilize three metrics for validation purposes: mutual correlation of the elements of the fading matrix $G$, mutual information, ${ }^{4}$ and the 2-D angular power spectrum. First, model parameters are extracted from the measurements and synthetic channel realizations are created by means of the parameterized models. The size of the synthetic ensemble is the same as of the measured ensembles. Second, mutual information values and angular power spectra are calculated from measured and synthetic MIMO channel impulse responses and compared to each other.

When calculating the mutual information of MIMO channels, we assume that the transmitter has no knowledge about the channel at all. Thus, the mutual information is given by [1], [2]

$$
I=\mathrm{E}\left\{\log _{2}\left[\operatorname{det}\left(\boldsymbol{I}_{M_{\mathrm{A}}}+\frac{\mathrm{SNR}}{M_{\mathrm{B}}} \boldsymbol{H} \boldsymbol{H}^{\mathrm{H}}\right)\right]\right\}
$$

where SNR denotes the signal-to-noise ratio, and the average energy of the channel matrix entries $h_{m, n}$ is normalized to unity. The expectation operation is performed with respect to measured channel realizations or fading realizations of the random matrix $\boldsymbol{G}$ when applying (5), (3), or (2). For the following evaluations, the receive SNR was set to $20 \mathrm{~dB}$.

\section{A. Extraction of Model Parameters From Measurements}

The correlation matrices for the "Kronecker model" are given by $\boldsymbol{R}_{\mathrm{A}}$ and $\boldsymbol{R}_{\mathrm{B}}$, where expectation is performed with respect to measured channel realizations. As basis matrices of the new model, we utilize the eigenbases $\boldsymbol{U}_{\mathrm{A}}$ and $\boldsymbol{U}_{\mathrm{B}}$ of these correlation matrices obtained by eigendecomposition. For the "virtual channel representation," the basis matrices $\boldsymbol{A}_{\mathrm{A}}$ and $\boldsymbol{A}_{\mathrm{B}}$ are constructed as explained in [16].

An estimate of the coupling matrix $\boldsymbol{\Omega}$ of the new model is obtained from the measured impulse responses $\boldsymbol{H}$

$$
\boldsymbol{\Omega}=\mathrm{E}_{\boldsymbol{H}}\left\{\left(\boldsymbol{U}_{\mathrm{A}}^{\mathrm{H}} \boldsymbol{H} \boldsymbol{U}_{\mathrm{B}}^{*}\right) \odot\left(\boldsymbol{U}_{\mathrm{A}}^{\mathrm{T}} \boldsymbol{H}^{*} \boldsymbol{U}_{\mathrm{B}}\right)\right\}
$$

which is equivalent to a calculation via the full correlation matrix $\boldsymbol{R}_{\boldsymbol{H}}$ (1)

$$
\omega_{m, n}=\left(\boldsymbol{u}_{\mathrm{B}, n} \otimes \boldsymbol{u}_{\mathrm{A}, m}\right)^{\mathrm{H}} \boldsymbol{R}_{\boldsymbol{H}}\left(\boldsymbol{u}_{\mathrm{B}, n} \otimes \boldsymbol{u}_{\mathrm{A}, m}\right) .
$$

By using the predefined basis matrices $\boldsymbol{A}_{\mathrm{A}}$ and $\boldsymbol{A}_{\mathrm{B}}$ instead of the eigenbases, the coupling matrix $\boldsymbol{\Omega}_{\mathrm{virt}}$ of the "virtual channel representation" is estimated analogously.

\section{B. Indoor Measurements}

Channel matrices were measured in the Electrical Engineering Building on the Vienna University of Technology Campus at $5.2 \mathrm{GHz}$ [3]. The transmitter consisted of a positionable monopole antenna on a grid of $20 \times 10$ positions with an interelement spacing of half the wavelength. The receiver employed a ULA of eight directional printed dipoles having an

\footnotetext{
${ }^{4}$ In the literature, the mutual information according to (7) is also referred to as MIMO channel capacity for unknown channel at the transmitter.
} 
(a)

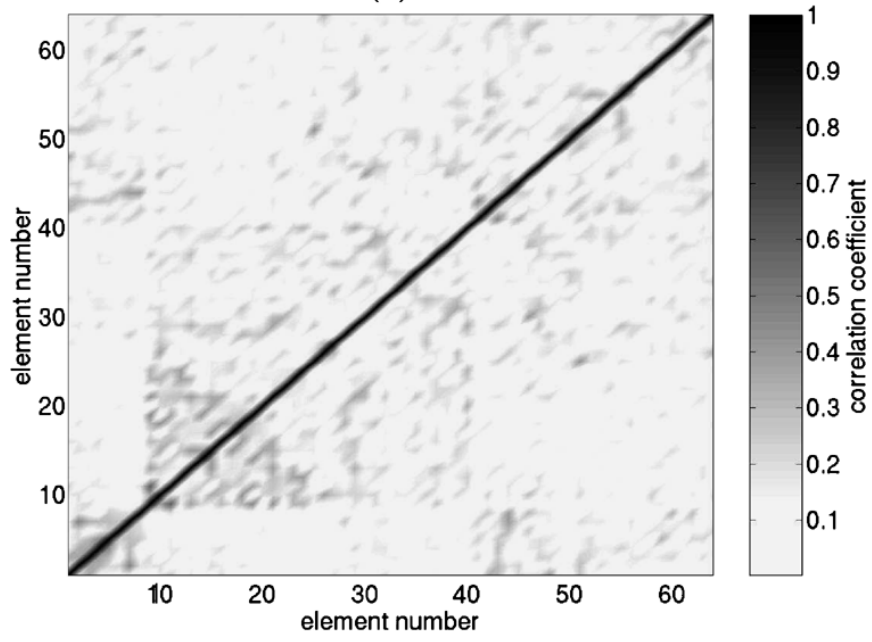

(b)

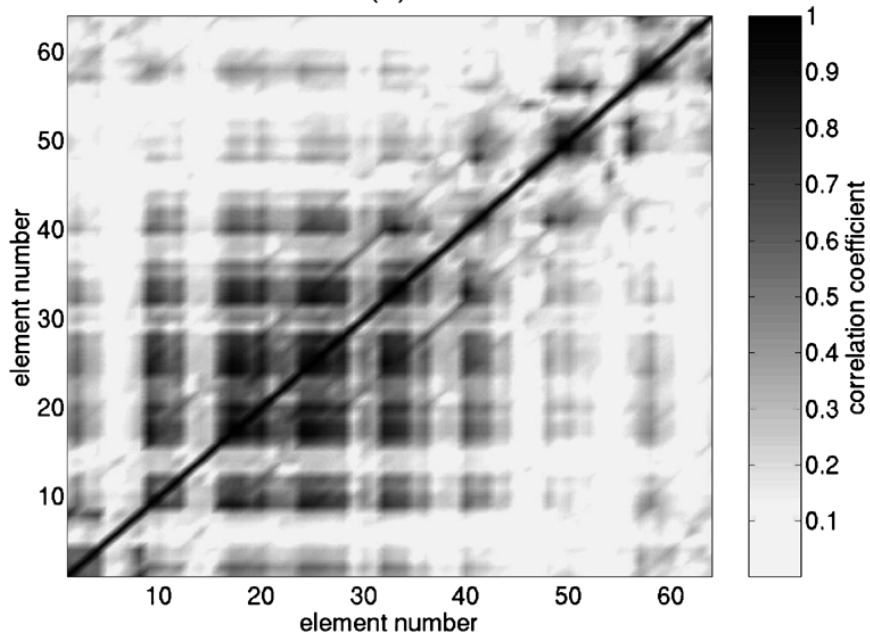

Fig. 3. Absolute values of the correlation coefficients of fading matrix elements for (a) new model and (b) "virtual channel representation."

interelement spacing of 0.4 wavelengths and a $3 \mathrm{~dB}$ beamwidth of $120^{\circ}$. The channel was probed at 193 equi-spaced frequency bins over $120 \mathrm{MHz}$ of bandwidth. The transmitter assumed a single fixed location in a hallway. The Rx array assumed many different locations in several offices connected to this hallway as well as three possible orientations. In total, 72 scenarios, i.e., Rx positions and orientations, were measured. For each scenario, 130 spatial realizations of an $8 \times 8$ channel matrix were formed by moving a virtual eight-element ULA over the $20 \times 10$ grid, yielding a total of $130 \cdot 193$ (space and frequency) realizations per scenario.

Fig. 3 illustrates the importance of choosing the right basis matrices by comparing the new model with the "virtual channel representation." From the measured data, we calculated the mutual correlation coefficients of all $8 \times 8$ fading amplitudes $\left(\boldsymbol{U}_{\mathrm{A}}^{\mathrm{H}} \boldsymbol{H} \boldsymbol{U}_{\mathrm{B}}^{*}\right)$ and $\left(\boldsymbol{A}_{\mathrm{A}}^{\mathrm{H}} \boldsymbol{H} \boldsymbol{A}_{\mathrm{B}}^{*}\right)$, respectively. In Fig. 3, the absolute values of the $64 \times 64$ correlation coefficients are depicted for (a) the new model and (b) the "virtual channel representation." Since the fading matrix $\boldsymbol{G}$ is modeled as i.i.d., the cross-correlation coefficients should be exactly zero for both models. The new model shows only a few and rather low

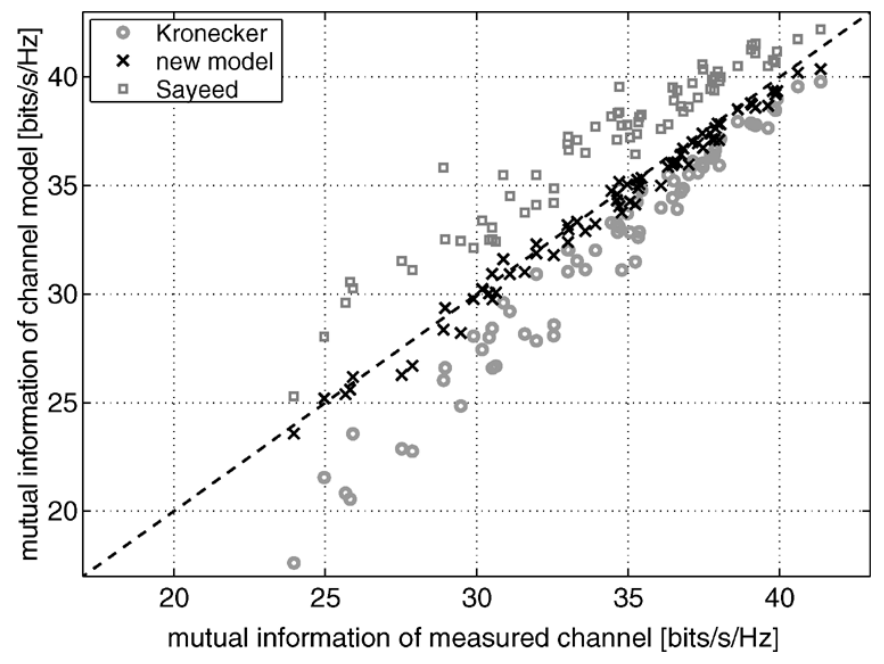

Fig. 4. Mutual information for each of the 72 measured indoor scenarios according to three different MIMO channel models versus measured mutual capacity.

correlation coefficients that are due to a slight noncompliance with assumption (4). On the other hand, the fixed bases of the "virtual channel representation" cause significant correlation values for a large portion of correlation coefficients.

In Fig. 4, we compare three different models: the "Kronecker model," the "virtual channel representation" (labeled as "Sayeed"), and the new model. The figure shows the modeled mutual information versus the measured mutual information for each of the 72 scenarios by means of a scatter plot. Each data point in the figure corresponds to a specific model and a specific scenario. The identity line (dashed) indicates the points of no modeling error. Obviously, the "Kronecker model" underestimates the mutual information and the "virtual channel representation" tends to overestimate. The new model shows a rather good match between measured and modeled mutual information.

Fig. 5 gives a clue why the "Kronecker model" underestimates mutual information but the new model does not. It shows the 2-D joint angular power spectrum for a single scenario, which was obtained by a 2-D Fourier transform. ${ }^{5}$ The measured spectrum (a) shows a clear linkage of specific direction of departure (DoD) to specific direction of arrival (DoA). The new model (b) changes the spectrum slightly because assumption (4) is not completely fulfilled. However, the linkage of DoDs to DoAs is preserved to a large extent. The "Kronecker model" (c), on the other hand, destroys the dependence of DoAs on DoDs and produces a 2-D spectrum that is a multiplication of two one-dimensional (1-D) spectra. This is a direct consequence of the separability assumption of the "Kronecker model," which forces the coupling matrix to be of rank one. The virtual channel representation (d) shows a very good fit to the measured spectrum. However, keep in mind that the power spectrum does not show any correlations between its peaks. While the peaks of the measured channel are uncorrelated, the virtual channel

\footnotetext{
${ }^{5}$ In order to calculate the 2-D power spectrum, the array geometries of both link ends have to be known.
} 
(a)

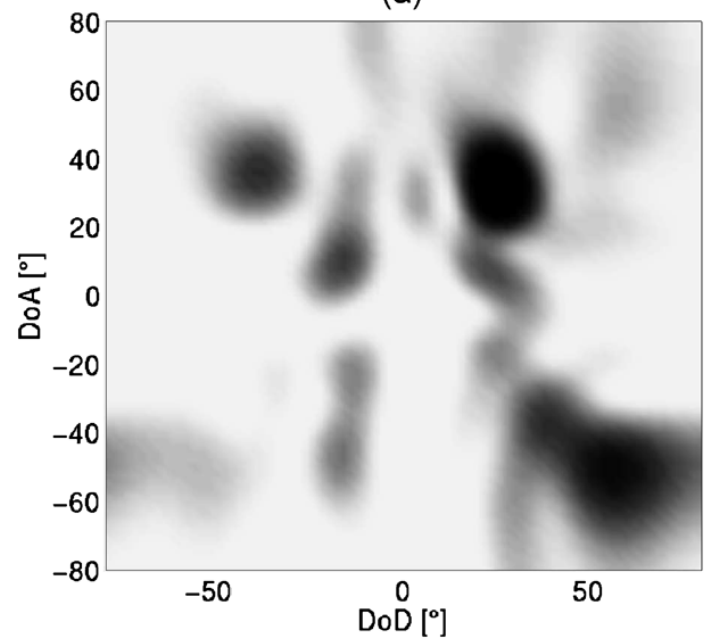

(c)

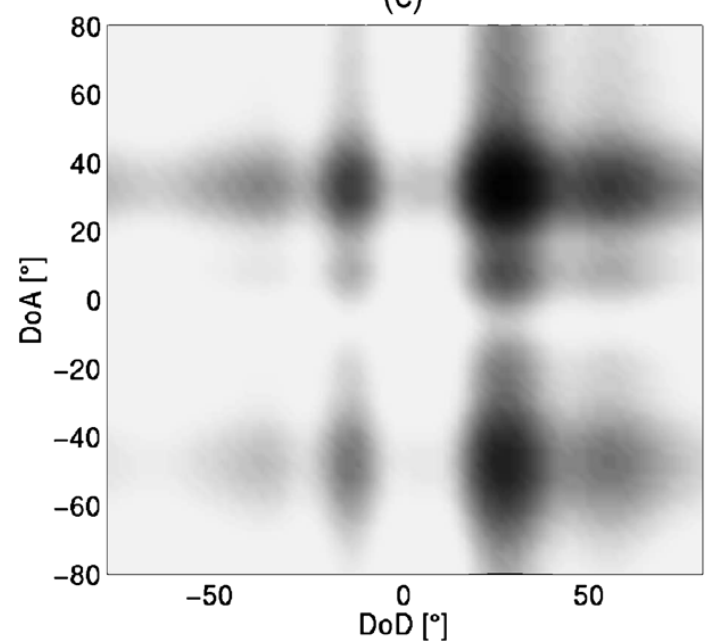

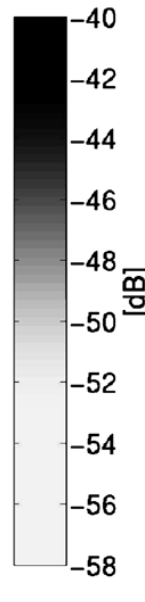
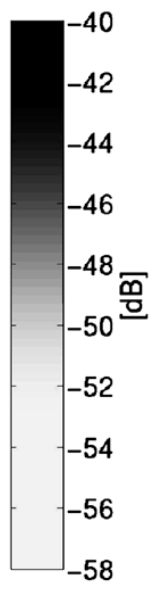

(b)

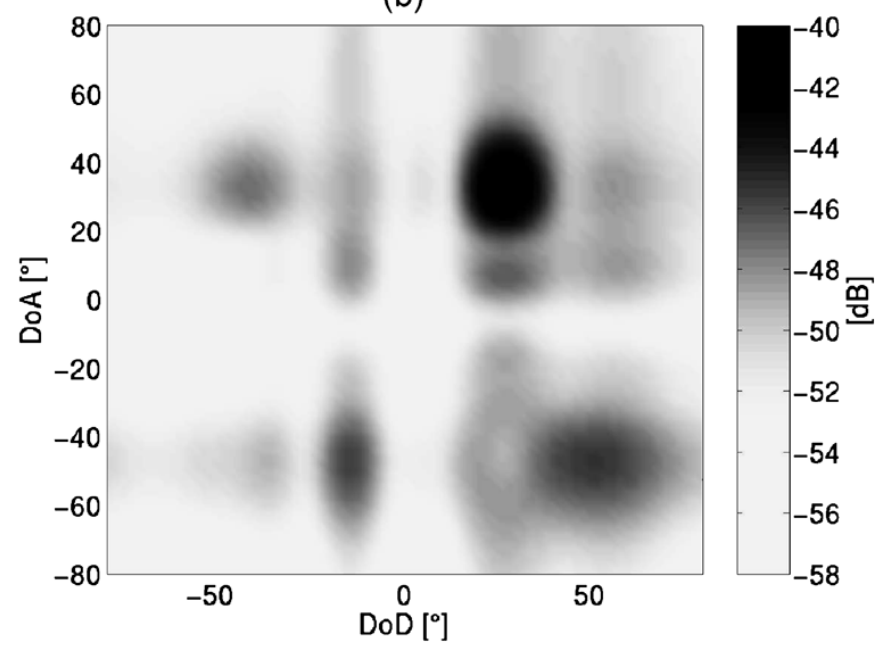

(d)

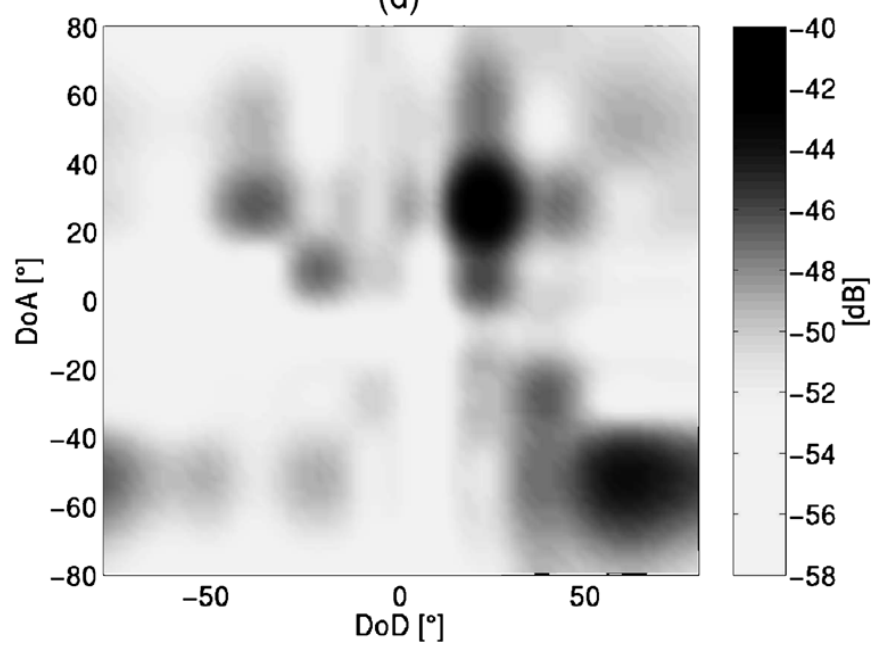

Fig. 5. (a) 2-D angular power spectrum of one measured indoor scenario. (b) Result of the new model. (c) Result of the "Kronecker model." (d) Result of the "virtual channel representation."

representation introduces artefact correlations (cf. Fig. 3 and discussion thereof), which cannot be seen in Fig. 5.

We want to point out that Fig. 5 is not representative for all scenarios of the indoor measurement campaign. Although the proposed model always performs better than the Kronecker model, the performance difference is in some cases minor, i.e., also the proposed model occasionally happens to produce a rather blurred version of the power spectrum. The virtual channel representation shows a rather good fit for all cases.

\section{Outdoor Measurements}

The measurements were carried out at the village of Weikendorf [25], a suburban area in Austria. 193 equi-spaced frequency bins over a bandwidth of $120 \mathrm{MHz}$ were measured at $2 \mathrm{GHz}$. The receiver was connected to a ULA of eight directional patch elements with an interelement spacing of half the wavelength, and was mounted at a height of approximately $20 \mathrm{~m}$. The transmitter, a uniform circular array of 15 monopoles with a radius of 1.03 wavelengths, was placed on a trolley at a height of about $1.5 \mathrm{~m}$ above ground and moved through streets at speeds of about 3 to $6 \mathrm{~km} / \mathrm{h}$ [25].
For the evaluations, MIMO snapshots with a time resolution of $84 \mathrm{~ms}$ were used. This is equivalent to a resolution in space of about half the wavelength. We grouped five consecutive time snapshots to one "scenario." In total, we get 50 different scenarios along the measurement route of the transmitter. The total set of channel samples per scenario was formed by 193 equi-spaced frequency samples within the measurement bandwidth times the five temporal snapshots. The normalization was done separately for each scenario. Because the steering vectors of the applied circular array have very low side-lobe suppression, we did not apply the "virtual channel representation."

Fig. 6 compares the predicted mutual information values of the "Kronecker model" and the new model with measured data of the outdoor scenario. The movement along the measurement route is projected onto the $x$-axis; the $y$-axis shows the mutual information at a specific location of the route. Again, we can see clearly that the "Kronecker model" underestimates the mutual information. The new model shows significantly less modeling error. However, for this measurement campaign, the new model tends to slightly overestimate the mutual information. 


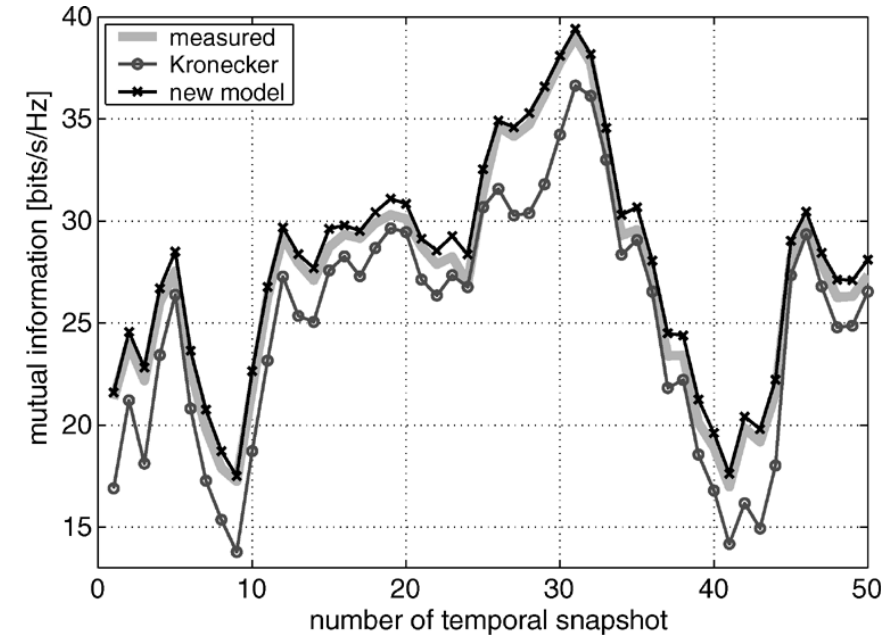

Fig. 6. Mutual information along the outdoor measurement route for the measured data, the new model, and the "Kronecker model."

\section{CONCLUSiON}

We presented a novel stochastic model for MIMO radio channels that is based on the joint correlation properties of both link ends. The necessary and sufficient condition for the proposed model to hold is that the eigenbasis at the receiver is independent of the transmit weights, and vice versa. The required model parameters are the eigenbasis at the receiver, the eigenbasis at the transmitter, and a coupling matrix that specifies how much energy is transported from each transmit eigenmode to each receive eigenmode on average. The number of elements in this coupling matrix is $M_{\mathrm{A}} \cdot M_{\mathrm{B}}$, as compared to $M_{\mathrm{A}}+M_{\mathrm{B}}$ receive and transmit eigenvalues of the "Kronecker model," which is not able of modeling general MIMO channels accurately. For MIMO channels whose correlation properties are truly separable into $\mathrm{Rx}$ and Tx components, the new model reduces to the "Kronecker model."

As a reward for the increased number of modeling parameters, the proposed model, besides being able to predict measured mutual information values, shows an improved capability of rendering 2-D power spectra of measured MIMO channels, linking stochastic MIMO channel modeling to physical wave propagation. This link to physical wave propagation is also provided by the "virtual channel representation," which, in contrast to our proposed model, approximates the true eigenbases by fixed and predefined DFT matrices. The different choice of basis matrices has some implications. The proposed model decorrelates the channel coefficients in the eigendomain as much as possible (within the structural restrictions of the model), whereas the "virtual channel representation" shows significant residual correlations for finite antenna arrays. While the proposed model renders the mutual information of measured channels almost exactly, the "virtual channel representation" provides more accurate estimates of 2-D angular power spectra. The "virtual channel representation," whose application is restricted to ULAs, provides a simple and intuitive relation to physical directions and propagation environments. Moreover, the predefined DFT matrices do not have to be recalculated for each environment. On the other hand, the proposed model adapts the eigenbases to the individual environment and radio setup, which allows for the modeling of arbitrary antenna elements or array geometries. Specifically, the proposed model can also easily deal with dual-polarized antenna elements.

\section{APPENDIX \\ PROOF OF THEOREM 1}

Consider a MIMO channel matrix that is modeled as

$$
\boldsymbol{H}=\boldsymbol{B}_{\mathrm{A}}\left(\left(\sum_{k=1}^{K} \tilde{\boldsymbol{\lambda}}_{\mathrm{A}, k} \tilde{\boldsymbol{\lambda}}_{\mathrm{B}, k}^{\mathrm{T}}\right) \odot \boldsymbol{G}\right) \boldsymbol{B}_{\mathrm{B}}^{\mathrm{T}}
$$

where $\boldsymbol{B}_{\mathrm{A}}$ and $\boldsymbol{B}_{\mathrm{B}}$ are unitary matrices, $\boldsymbol{G}$ is an i.i.d. random matrix with zero mean and unit variance elements, and the vectors $\tilde{\boldsymbol{\lambda}}_{\mathrm{A}, k}$ and $\tilde{\boldsymbol{\lambda}}_{\mathrm{A}, k}$ are real valued.

With $\boldsymbol{\Lambda}_{\mathrm{A}, k} \triangleq \operatorname{diag}\left(\tilde{\boldsymbol{\lambda}}_{\mathrm{A}, k}\right)^{2}, \boldsymbol{\Lambda}_{\mathrm{B}, k} \triangleq \operatorname{diag}\left(\tilde{\boldsymbol{\lambda}}_{\mathrm{B}, k}\right)^{2}$, and $\boldsymbol{Q}_{\mathrm{B}}^{\prime} \triangleq$ $\boldsymbol{B}_{\mathrm{B}}^{\mathrm{T}} \boldsymbol{Q}_{\mathrm{B}} \boldsymbol{B}_{\mathrm{B}}^{*}$, the elements of the matrix

$$
\boldsymbol{\Xi}_{k} \triangleq \mathrm{E}_{\boldsymbol{H}}\left\{\boldsymbol{\Lambda}_{\mathrm{A}, k}^{\frac{1}{2}} \boldsymbol{G} \boldsymbol{\Lambda}_{\mathrm{B}, k}^{\frac{1}{2}} \boldsymbol{Q}_{\mathrm{B}}^{\prime} \boldsymbol{\Lambda}_{\mathrm{B}, k}^{\frac{1}{2}} \boldsymbol{G}^{\mathrm{H}} \boldsymbol{\Lambda}_{\mathrm{A}, k}^{\frac{1}{2}}\right\}
$$

can be calculated as

$$
\begin{gathered}
{\left[\boldsymbol{\Xi}_{k}\right]_{m, m^{\prime}}} \\
=\mathrm{E}_{\boldsymbol{H}}\left\{\sum_{n=1}^{M_{\mathrm{B}}} \sum_{n^{\prime}=1}^{M_{B}}[\boldsymbol{G}]_{m, n}\left[\tilde{\boldsymbol{\lambda}}_{\mathrm{A}, k}\right]_{m}\left[\tilde{\boldsymbol{\lambda}}_{\mathrm{B}, k}\right]_{n}\left[\boldsymbol{Q}_{\mathrm{B}}^{\prime}\right]_{n, n^{\prime}}\right. \\
\left.\cdot\left[\tilde{\boldsymbol{\lambda}}_{\mathrm{B}, k}\right]_{n^{\prime}}\left[\tilde{\boldsymbol{\lambda}}_{\mathrm{A}, k}\right]_{m^{\prime}}\left[\boldsymbol{G}^{*}\right]_{m^{\prime}, n^{\prime}}\right\} \\
= \begin{cases}{\left[\boldsymbol{\Lambda}_{\mathrm{A}, k}\right]_{m, m} \sum_{n=1}^{M_{\mathrm{B}}}\left[\boldsymbol{\Lambda}_{\mathrm{B}, k}\right]_{n, n}\left[\boldsymbol{Q}_{\mathrm{B}}^{\prime}\right]_{n, n},} & \text { if } m^{\prime}=m \\
0, & \text { else }\end{cases} \\
= \begin{cases}{\left[\boldsymbol{\Lambda}_{\mathrm{A}, k}\right]_{m, m} \operatorname{tr}\left(\boldsymbol{\Lambda}_{\mathrm{B}, k} \boldsymbol{Q}_{\mathrm{B}}^{\prime}\right),} & \text { if } m^{\prime}=m \\
0, & \text { else. }\end{cases}
\end{gathered}
$$

Now, the parameterized one-sided correlation matrix $\boldsymbol{R}_{\mathrm{A}, \boldsymbol{Q}_{B}}$ reads as

$$
\begin{aligned}
\boldsymbol{R}_{\mathrm{A}, \boldsymbol{Q}_{\mathrm{B}}} & =\boldsymbol{B}_{\mathrm{A}}\left(\sum_{k=1}^{K} \boldsymbol{\Xi}_{k}\right) \boldsymbol{B}_{\mathrm{A}}^{\mathrm{H}} \\
& =\boldsymbol{B}_{\mathrm{A}}\left(\sum_{k=1}^{K} \boldsymbol{\Lambda}_{\mathrm{A}, k} \cdot \operatorname{tr}\left(\boldsymbol{\Lambda}_{\mathrm{B}, k} \boldsymbol{B}_{\mathrm{B}}^{\mathrm{T}} \boldsymbol{Q}_{\mathrm{B}} \boldsymbol{B}_{\mathrm{B}}^{*}\right)\right) \boldsymbol{B}_{\mathrm{A}}^{\mathrm{H}} .
\end{aligned}
$$

The proof for $\boldsymbol{R}_{\mathrm{B}, \boldsymbol{Q}_{\mathrm{A}}}$ can be shown accordingly.

\section{ACKNOWLEDGMENT}

The authors would like to thank the Radio Communications Laboratory of Nokia Research Center in Helsinki for generously funding the research activities toward the presented MIMO channel model. Furthermore, they want to express gratitude to H. Hofstetter (Forschungszentrum Telekommunikation Wien, ftw) for supplying the outdoor measurement data, and T-Systems Nova GmbH for providing an eight-element ULA. 


\section{REFERENCES}

[1] I. E. Telatar, "Capacity of multi-antenna Gaussian channels," Bell Labs., Lucent Tech., Murray Hill, NJ, Oct. 1998, Tech. Memo.

[2] _ - "Capacity of multi-antenna Gaussian channels," Eur. Trans. Telecommun., vol. 10, no. 6, pp. 585-595, Nov./Dec. 1999.

[3] M. Herdin, H. Özcelik, H. Hofstetter, and E. Bonek, "Variation of measured indoor MIMO capacity with receive direction and position at $5.2 \mathrm{GHz}$," Electron. Lett., vol. 38, no. 21, pp. 1283-1285, Oct. 2002.

[4] J. P. Kermoal, L. Schumacher, K. I. Pedersen, P. E. Mogensen, and F. Frederiksen, "A stochastic MIMO radio channel model with experimental validation," IEEE J. Sel. Areas Commun., vol. 20, no. 6, pp. 12111226, Aug. 2002.

[5] D. P. McNamara, M. A. Beach, and P. N. Fletcher, "Spatial correlation in indoor MIMO channels," in Proc. IEEE Int. Symp. Personal, Indoor and Mobile Radio Communications (PIMRC), Lisbon, Portugal, 2002, vol. 1, pp. 290-294.

[6] K. Yu, M. Bengtsson, B. Ottersten, D. McNamara, P. Karlsson, and M. Beach, "Second order statistics of NLOS indoor MIMO channels based on $5.2 \mathrm{GHz}$ measurements," in Proc. Global Telecommunications Conf. (GLOBECOM), San Antonio, TX, 2001, vol. 1, pp. 156-160.

[7] C.-N. Chuah, D. N. C. Tse, J. M. Kahn, and R. A. Valenzuela, "Capacity scaling in MIMO wireless systems under correlated fading," IEEE Trans. Inf. Theory, vol. 48, no. 3, pp. 637-650, Mar. 2002.

[8] D. S. Shiu, G. J. Foschini, M. J. Gans, and J. M. Kahn, "Fading correlation and its effect on the capacity of multielement antenna systems," IEEE Trans. Commun., vol. 48, no. 3, pp. 502-513, Mar. 2000.

[9] H. Xu, D. Chizhik, H. Huang, and R. Valenzuela, "A wave-based wideband MIMO channel modeling technique," in Proc. IEEE Int. Symp. Personal, Indoor and Mobile Radio Communications (PIMRC), Lisbon, Portugal, Sep. 2002, vol. 4, pp. 1626-1630.

[10] K. I. Pedersen, J. B. Andersen, J. P. Kermoal, and P. E. Mogensen, "A stochastic multiple-input-multiple-output radio channel model for evaluation of space-time coding algorithms," in Proc. IEEE Vehicular Technology Conf. (VTC)-Fall, Boston, MA, Sep. 2000, vol. 2, pp. 893-897.

[11] D. Gesbert, H. Bölcskei, D. A. Gore, and A. J. Paulraj, "Outdoor MIMO wireless channels: Models and performance prediction," IEEE Trans. Commun., vol. 50, no. 12, pp. 1926-1934, Dec. 2002.

[12] K. Yu, M. Bengtsson, B. Ottersten, D. McNamara, P. Karlsson, and M. Beach, "A wideband statistical model for NLOS indoor MIMO channels," in Proc. IEEE Vehicular Technology Conf. (VTC)-Spring, Birmingham, AL, May 2002, vol. 1, pp. 370-374.

[13] M. T. Ivrlac, W. Utschick, and J. A. Nossek, "Fading correlations in wireless MIMO communication systems," IEEE J. Sel. Areas Commun., vol. 21, no. 5, pp. 819-828, Jun. 2003.

[14] H. Özcelik, M. Herdin, W. Weichselberger, J. Wallace, and E. Bonek, "Deficiencies of the 'Kronecker' MIMO radio channel model," Electron. Lett., vol. 39, no. 16, pp. 1209-1210, Aug. 2003.

[15] J. Wallace, H. Özcelik, M. Herdin, E. Bonek, and M. Jensen, "Power and complex envelope correlation for modeling measured indoor MIMO channels: A beamforming evaluation," in Proc. IEEE Vehicular Technology Conf. (VTC)—Fall, Orlando, FL, Oct. 2003, pp. 363-367.

[16] A. M. Sayeed, "Deconstructing multiantenna fading channels," IEEE Trans. Signal Process., vol. 50, no. 10, pp. 2563-2579, Oct. 2002.

[17] W. Weichselberger and H. Özcelik, "A novel stochastic MIMO channel model and its physical interpretation," in Proc. 7th COST-273 Meeting and Workshop, Paris, France, May 2003, TD(03)144, CD-ROM.

[18] J. Kotecha and A. M. Sayeed, "Optimal signal design for estimation of correlated MIMO channels," in Proc. IEEE Int. Conf. Communications (ICC), Anchorage, AK, 2003, vol. 5, pp. 3170-3174.

[19] A. Tulino, A. Lozano, and S. Verdú, "Capacity input covariance for correlated multi-antenna channels," in Proc. Allerton Conf., Monticello, IL, Oct. 2003, pp. 242-251.

[20] J. Kotecha and A. M. Sayeed, "Transmit signal design for optimal estimation of correlated MIMO channels," IEEE Trans. Signal Process., vol. 52, no. 2, pp. 546-557, Feb. 2004.

[21] R. Vaughan and J. B. Andersen, Channels, Antennas, and Propagation for Mobile Communications. London, U.K.: IEE, 2003.

[22] D. Chizhik, G. J. Foschini, M. J. Gans, and R. A. Valenzuela, "Keyholes, correlations, and capacities of multielement transmit and receive antennas," IEEE Trans. Wireless Commun., vol. 1, no. 2, pp. 361-368, Apr. 2002.

[23] P. Almers, F. Tufvesson, and A. F. Molisch, "Measurement of keyhole effects in a wireless multiple-input multiple-output (MIMO) channel," IEEE Commun. Lett., vol. 7, no. 8, pp. 373-375, Aug. 2003.
[24] K. Liu, V. Raghavan, and A. M. Sayeed, "Capacity scaling and spectral efficiency in wide-band correlated MIMO channels," IEEE Trans. Inf. Theory, vol. 49, no. 10, pp. 2504-2526, Oct. 2003.

[25] H. Hofstetter, I. Viering, and W. Utschick, "Evaluation of suburban measurements by eigenvalue statistics," in Proc. 1st Int. COST 273 Workshop, Espoo, Finland, May 2002, CD-ROM.

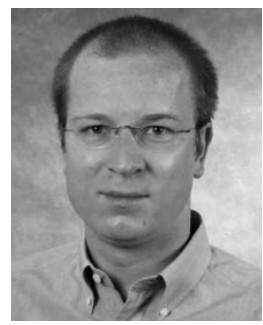

Werner Weichselberger was born in Vienna, Austria, in 1973. He received the degree (with highest honors) in electrical engineering from Vienna University of Technology (TU Wien), Vienna, in 1999, after writing the diploma thesis in Munich under the direction of Prof. Utschick about neural networks, and the Dr.Techn. degree (with highest honors) from Vienna University of Technology, in January 2004

In 1999, he joined the mobile communications research group of Prof. Bonek at Vienna University of Technology, where he was engaged in a tight cooperation with Nokia Research Center, Helsinki, Finland. His research concentrated on link-level algorithms for Global System for Mobile Communications (GSM) and Universal Mobile Telecommunications System (UMTS), spatiotemporal signal processing, and spatial modeling of radio channels. In 2004, he co-founded the company Woolf Solutions, Vienna, Austria, which provides IT consulting and algorithm design.

Mr. Weichselberger was an active Member of the European Research Initiatives COST 260 and COST 273.

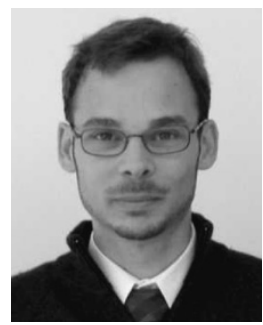

Markus Herdin received the Dipl.Ing. degree in electrical engineering and the Ph.D. degree (with highest honors) both from the Technische Universität Wien (TU Wien), Vienna, Austria, in 2001 and 2004, respectively.

From 2000 to 2004, he was a member of the mobile communications group of Prof. Bonek at the Institute of Communications and Radio-Frequency Engineering, TU Wien. $\mathrm{He}$ is currently with DoCoMo Communications Laboratories Europe $\mathrm{GmbH}$, Munich, Germany. Former research areas covered multiuser detectors for Universal Mobile Telecommunications System (UMTS), mutual interference of Bluetooth and wireless local area network (WLAN), multiple-input multiple-output (MIMO) channel measurements, and MIMO channel characterization. His current research focus lies on multihop transmission for $4 \mathrm{G}$ systems.

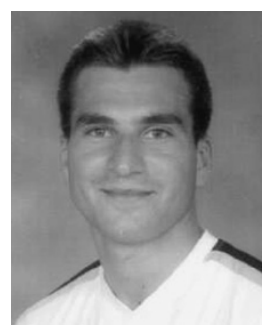

Hüseyin Özcelik (SM'01) was born in Vienna, Austria, in 1976. He received the Dipl.Ing. degree (with highest honors) in electrical engineering from the Technische Universität Wien (TU Wien), Vienna, in 2001, and is currently working toward the Ph.D. degree at the Mobile Communications Group, Institut fur Nachrichtentechnik und Hochfrequenztechnik, TU Wien.

His main research areas are multiple-input multiple-output (MIMO) radio channel measurements, characterization, and modeling.

Mr. Ozcelik is an active Member of the European Research Initiative COST 273 


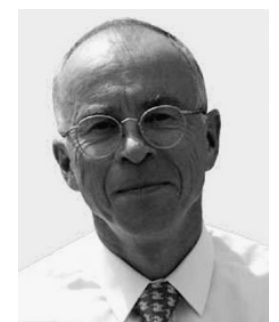

Ernst Bonek (M'73-SM'85) was born in Vienna, Austria, in 1942. He received the Dipl.Ing. and Dr.Techn. degrees (with highest honors) from Technische Universität Wien (TU Wien), Vienna.

In 1984, he was appointed Full Professor of Radio Frequency Engineering at TU Wien, from which he retired in May 2004. His field of interest is mobile communications at large. Recent contributions concern smart antennas, the characterization of mobile radio channels, and advanced antennas and receiver designs. His group pioneered three-dimensional (3-D) superresolution measurements of the urban mobile radio channel, the "double-directional" viewpoint of the mobile radio channel, and propagationbased MIMO channel models. Previous fields of research were semiconductors, microwaves, optical communications, and intersatellite links. Altogether, he (co)authored some 170 journal and conference publications. He holds several patents on mobile radio technology. He coauthored the book Data Transmission over GSM and UMTS (Springer-Verlag) and coedited, with the late Jean-Claude Bic, Technology Advances of UMTS (Hermes Scientific Publications).

Dr. Bonek served as the Chairman of the IEEE Austria Section from 1985 to 1990. From 1991 to 1994, he was a Council Member of the Austrian Science Fund, acting as Speaker for engineering sciences. From 1996 to 1999, he served on the Board of Directors of the reorganized Post and Telekom Austria. He participated in the European Research Initiative COST 259 as Chairman of the working group on Antennas and Propagation, and continues to serve in this position in COST 273. In URSI, he was the Chairman of Commission C "Signals and Systems" between 1999 and 2002. He was the initiator of Forschungszentrum Telekommunikation Wien ( $\mathrm{ftw}$ ), a public-private partnership for telecommunications research in Vienna. 